\title{
Simulation of Excavator Bucket Pressuring Through Finite Element Method
}

\author{
Sumar Hadi Suryo ${ }^{a^{*}}$, Athanasius Priharyoto Bayuseno a, Jamari a, Gilang Ramadhan ${ }^{\text {a }}$ \\ ${ }^{a}$ Department of Mechanical Engineering, University of Diponegoro, Semarang, Indonesia.
}

Received 17 January 2018; Accepted 12 March 2018

\begin{abstract}
Excavator bucket tool is one of the most important parts of an excavator. It is made of steel mixture. It is commonly equipped with protruding teeth on its cutting side to breakdown hard materials and also to prevent dryness and damage of the bucket. Excavator bucket tooth must have supporting geometrical shape to penetrate and to endure the digging process on the ground, gravels, stones, or any other abrasive field. It is because of the field's natural characteristic when the bucket tool grinds the material. Mixed iron is common to be used as excavator bucket tool's material because it is easy to get and economic. High hardness value is also needed on the surface that transports hard material such as mining equipment. Therefore, an accurate analysis should be done to determine the suitable material on this field. Design and analysis were done by using Computer-Aided Engineering (CAE) Abaqus 6.10 application to get the maximum tension as the result of loading. Analysis process to get the tension was done by adding $8285.06 \mathrm{~N}$ weight forces in static condition with the angle of $32^{\circ}$ to the horizon. From the analysis it could be known that maximum tension experienced by excavator bucket tooth is 209.3 MPa, and it is still below the Maximum Equivalent von Mises stress so the design could be categorized as safe.
\end{abstract}

Keywords: Abaqus 6.10; Bucket Tooth; Design; Excavator; Finite Element; Von Mises.

\section{Introduction}

Knowledge and technology are growing rapidly along with the developing era, supported by more intelligent human resources. The development of knowledge is needed because of the strict of competition and increase of society's needs. The experts are trying to create and find a new method to fulfil those needs. The educational practitioners are doing the same thing to be able to add new discourses and atmosphere needed by market. We are welcoming the global market in which demand competition without paying attention of whom, how, and where. Meanwhile, the most important thing is to face and win the competition in the global market itself. The rapid development of all living aspects and the increasing complexity of human's needs motivate people to create sophisticated equipment. That equipment can help to ease the work processes, minimize working time of building big projects such as highways, sky scrapers, fly over, airports, and etc. Therefore, we need an equipment which has the ability to do hard works such as digging ground, loading the ground, and many others with little time and high efficiency.

One of the heavy equipment which is commonly used in digging is excavator. Excavator is heavy equipment used in construction and mining industries to excavate holes and build foundations and other things. Excavator bucket is made of solid steel and is generally equipped with protruding teeth on its cutting side to breakdown hard material and prevent wear and damage of bucket. Excavator bucket teeth must have supporting loading capacity of materials such as wet ground, rocks and abrasive field caused by the nature of the ground when the bucket teeth breakdowns the material. Alloy steel is commonly used to make excavator bucket teeth [1].

\footnotetext{
* Corresponding author: sumarhs.undip@gmail.com

doil http://dx.doi.org/10.28991/cej-0309107
}

$>$ This is an open access article under the CC-BY license (https://creativecommons.org/licenses/by/4.0/).

(C) Authors retain all copyrights. 
Direct contact of metal components and the ground requires an alloy that has the characteristic of toughness and high abrasive resistance. High hardness value is also required on the surface that loads moving materials and even hard materials to manufacture grinding equipment for mining industry. In an excavator, the digger and lifter part is called an excavator bucket. The tip part of the bucket which plays the claw role is called bucket teeth which are the most likely part to fail when the excavator is operated [2]. The commonly used material of bucket teeth is mild carbon steel with a $\mathrm{C}$ content of about $0.33 \%-0.5 \%$ so it seems that the material is unable to withstand the loading and hardness of the material imposed on the excavator bucket teeth [3]. Moreover, it is mostly found that there are many bucket teeth excavators experiencing abrasion or even broken while working on a pretty heavy work in the field [4].

The inequality shape and composition of bucket teeth materials will lead to poor excavation process. In addition, it may cause high wear on the bucket teeth, decrease in project timeliness, cost, and productivity [5]. Generally, bucket teeth design is based on the use of bucket teeth on soft or hard media. The strength of the bucket teeth is obtained from the combination of material composition and the treatment during the manufacturing process [6].

Bucket teeth are part of the excavator that is frequently replaced. It is due to the expiration of bucket teeth which frequently causes failures when the excavator bucket makes penetration to the ground. The most frequent examples of failures of bucket teeth are wear and tear, crooked, cracked and broken due to abrasion on its sides. The failure of bucket teeth failure is commonly caused by several factors such as soil type, penetration velocity, penetration angle, and the material the bucket teeth is made of. For the sake of more efficient use, structural analysis is important to perform during the excavation, one of which is the analysis of the strength distribution of bucket teeth. It is performed to prevent failure of the bucket teeth and to prolong its lifetime in order to reduce the cost of excavator maintenance [7].

Zi-Qiang Fang et al. [8] conducted a research on the analysis of contact force of excavator bucket in the excavation process. By using DEM, different variation of excavation rates for excavation process of excavator bucket, pitch variation on teeth and variation on teeth angle, the results showed that Fnmax and Ftmax of the teeth and the increase of cutting angles by the increase of excavation rates can lead to the increase of wear probability to the teeth and cutting angles in the excavation process.

Jin Chen et al. [9] conducted a research on the methodology to characterize excavation performance of hydraulic excavator with uncertain consideration during the excavation process. The natural variability in the field and the difference in operating forces is a significant source of uncertainty. The most probable direction interval of excavation resistance that is obtained from the experimental data is used to measure the contribution of the uncertain medium to the direction variation of excavation resistance. By considering different operating styles, for each excavation point, the excavation angle is discretized by a certain step and the inverse kinematics approach is taken to obtain the appropriate manipulator configuration to each incremental step from the excavation angle.

Patel et al. [10] consider backhoe kinematics, differential motion, static and dynamic model of backhoe mechanism to develop development of generalized breakout and digging force model. There is no need to analyze every position and orientation (collectively known as the configuration) of the mechanism from the available breakout and digging forces, in static analysis one configuration of the mechanism has to be decided first for which the analysis is to be carried out. From all the configurations, the maximum breakout force condition is the most critical one as it produces the highest breakout force, and thus for this condition the force analysis is done, and will be used as a boundary condition for static FEA [10].

The analysis of this research used finite element method. Finite Element Method (FEM) is a numerical procedure that can be used to solve problems in the engineering field including stress analysis on the structure, personal frequency and its shape mode, heat transfer, electromagnetic and fluid flow. This method is used on engineering problems where the exact solution or analytical solution cannot solve it. The essence of the finite element method is to divide an object to be analyzed into several parts in the finite amount. These parts are called elements in which each element to another are associated with nodal titles. Thus, a mathematical equation is formulated to be the representation of the object [11]

\section{Material and Method}

\subsection{Bucket Tooth}

Bucket teeth (teeth or so-called bucket nails) are part of the bucket that frequently experience wear and tear when the excavator is operated because this part makes direct contact with the medium under the work by using the excavator. Inappropriate bucket teeth and composition of bucket teeth material will lead to a poor excavation process and also causing high wear on the bucket teeth, loss in time and cost and decrease in productivity [12]. 
Table 1. The Shape of Bucket Teeth

\begin{tabular}{|c|c|c|}
\hline Bucket teeth & Description & Figure \\
\hline Standard & It is used in loading, hauling, excavating works. Its wear rate is high. & \\
\hline Rock Chisel & $\begin{array}{l}\text { It is used for general excavation work with more abrasive materials. It has } \\
\text { wear and tear age of 3-5 times bigger than other shapes of teeth. }\end{array}$ & \\
\hline Rock Penetration & It is on work in rocky media that requires high penetration. & \\
\hline Unitooth & It is used in light to moderate work. & \\
\hline Penetration & $\begin{array}{l}\text { It is used for works that require high penetration. It is commonly used on } \\
\text { clay media. }\end{array}$ & \\
\hline
\end{tabular}

Flare It is used on excavators that have large transport capacity.

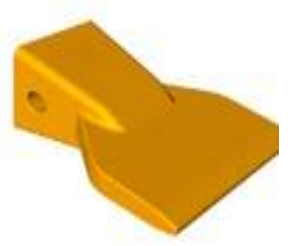
Tiger provides maximum penetration and is used for cohesive material. It is mounted in the middle side along with twin tiger on both sides of the bucket.

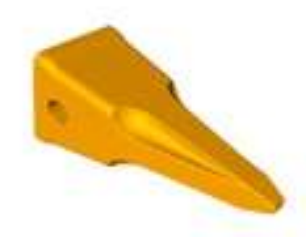

Twin Tiger

The material is more wear resistant. Its strength is great and may increase the fracture ability. It is usually mounted on both sides of the bucket.

Dimensional size of bucket tooth is needed in this research. Before it could be simulated, the bucket tooth should be built in three dimensional models first by using computer assisted software. The first thing to do is to get the whole dimensional size of the bucket tooth by direct measurement. And then, from that dimensional size, three dimensional model of bucket tooth can be made. 


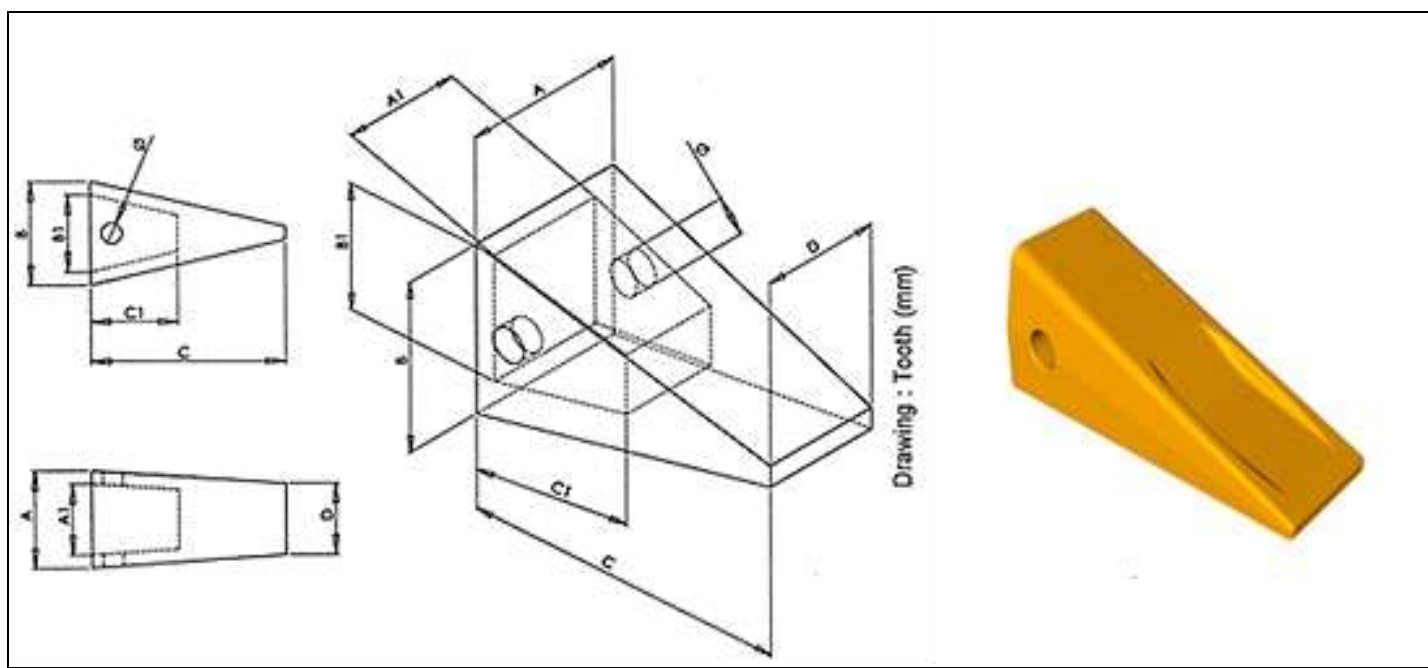

Figure 1. Bucket tooth [6]

Table 2. Bucket tooth size

\begin{tabular}{cccccccc}
\hline \multicolumn{7}{c}{ Dimensional Size (mm) } \\
\hline A & B & C & D & A1 & B1 & C1 & ø \\
\hline 98 & 102 & 220 & 76 & 75 & 84 & 95.3 & 31.8 \\
\hline
\end{tabular}

\subsection{Software Assisted Three Dimensional Modeling}

Loading simulation needs to be done by using computer software so that the work can be easier and more accurate. In this research, the Computer-Aided Engineering (CAE) used was Abaqus 6.10. By the usage of this software, all of the spots of bucket tooth can be analyzed with certain loading.

The type of bucket tooth modeled for the simulation process is standard bucket tooth. Standard type was chosen because of its wide usage and is commonly used in construction works. This bucket tooth was modeled resembling to its actual shape and size. Modeling was done by using $\mathrm{CAD}$ software. The design of bucket tooth was made after getting the dimensional data such as length, width, and height of bucket tooth from the measurement. The overall dimensional size of bucket tooth could be seen in Table 3 .

Table 3. Dimensional size of standard bucket tooth

\begin{tabular}{cc}
\hline Model & Standard Bucket Tooth \\
\hline Length & $220 \mathrm{~mm}$ \\
\hline Width & $98 \mathrm{~mm}$ \\
\hline Height & $102 \mathrm{~mm}$ \\
\hline Surface Area & $89576.48 \mathrm{~mm}^{2}$ \\
\hline Volume & $595213.17 \mathrm{~mm}^{3}$ \\
\hline
\end{tabular}

After knowing the fixed dimension of bucket tooth's geometry, design process was done by using SolidWorks software. Figure 3 shows the design of bucket tooth that was made. 


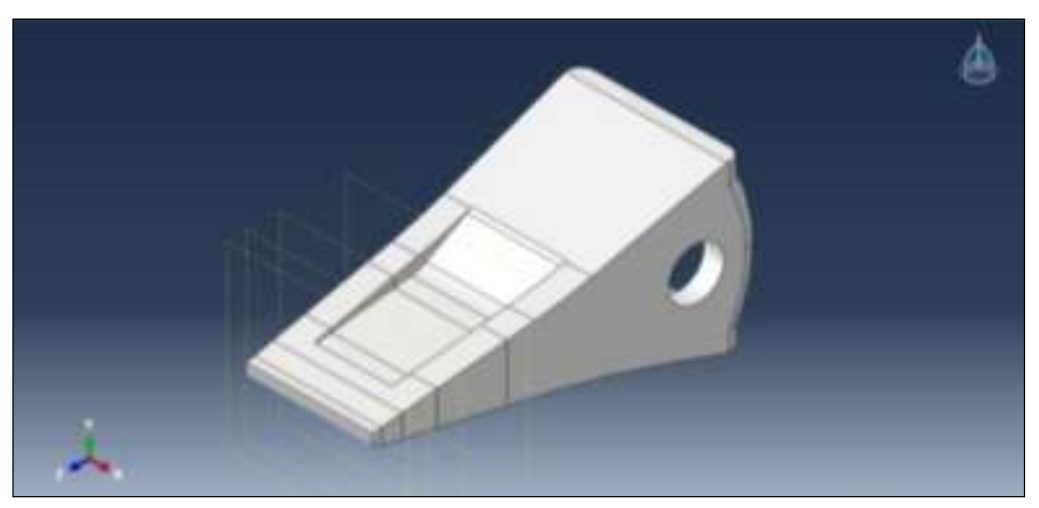

Figure 2. Three dimensional modeling of bucket tooth

In this research, the type of excavator used was Komatsu PC200, which is 20 tons mass operation excavator commonly used in construction's digging process.

\subsection{Material}

The components of excavator tooth were made of materials that fulfill the functional needs of each components. Materials used in those components should be suitable with the operational forces of the excavator tooth. The properties of those materials can be found in ASME (American Society of Mechanical Engineering) and the material composition can be seen in ASTM (American Standard Testing and Material). The material specification data obtained was then inserted to the software analysis, so that the data obtained from simulation is close to the real condition.

Bucket tooth was made from steel combined with iron, carbon, manganese, silisium, and other elements as mixture or residual. The mechanical properties of steel is highly depending on its carbon content. More carbon content in the steel will increase its hardness value and its endurance towards worn-out. Sometimes, steel that will made to be bucket tooth does not have enough hardness. Thus, hardening process should be done. By doing hardening, a higher hardness properties will be obtained. The higher the hardness value, the lower the tenacity property in which steel can be brittle. Since that steel is not quite good for use, therefore, after hardening process, tempering process is always done next [1].

Tempering is a process in which steel, which has been hardened, is heated again on certain temperature and hold for certain time to remove or to decrease remaining stress and to return a part of its tenacity and its strength. The return of it is obtained by sacrificing a part of strength and hardness got from hardening process.

Generally, bucket tooth making process was done by using Austempered Ductile Iron (ADI), Casting and Forging. In process of Austempered Ductile Iron (ADI), it obtained bucket tooth which has medium and high impact endurance. ADI tooth mainly is cast tooth with different chemical treatment, tough steel which was given heat treatment was in austempering. Casting process resulted bucket teeth with high strength and deep-hardening tenacity and had a very good strength for use and abrasion. While, in foCastinrging process, there was chrome-moly alloy steel with continuous fiber structure and grain of steel which gave strength raising by keep maintaining its hardness value. Bucket tooth from forging result has good tensile strength value as well as good tenacity value [6].

For material use in mining industry, alloy from Chromium, Niobium, vanadium and boron low usage of time [13].

This research uses bucket tooth from casting process in which material used was included into medium carbon steel category. Specification of material used to make bucket tooth is shown on Table 4 below.

Table 4. Bucket tooth material composition

\begin{tabular}{ccccccccccc}
\hline \multicolumn{10}{c}{ Chemical Composition (\%) } \\
\hline $\mathbf{C}$ & $\mathbf{S i}$ & $\mathbf{M n}$ & $\mathbf{P}$ & $\mathbf{S}$ & $\mathbf{C u}$ & $\mathbf{N i}$ & $\mathbf{C r}$ & $\mathbf{M o}$ & $\mathbf{A l}$ \\
\hline 0.37 & 0.25 & 1.34 & 0.017 & 0.006 & 0.007 & 0.022 & 0.32 & 0.16 & 0.011 \\
\hline
\end{tabular}

Property of material used in bucket tooth modeling is attached on Table 5. Property of material was obtained from test result which has been done in laboratory test. 
Table 5. Properties material of bucket tooth [14]

\begin{tabular}{cc}
\hline Type of Material & AISI 1040 steel \\
\hline Elastic Modulus & $205000 \mathrm{MPa}$ \\
\hline Mass Density & $7845 \times 10^{-12}$ ton $/ \mathrm{mm}^{3}$ \\
\hline Poison ratio & 0.29 \\
\hline Yield Strength & $435 \mathrm{MPa}$ \\
\hline Tensile Strength & $670 \mathrm{MPa}$ \\
\hline
\end{tabular}

\subsection{Methods}

Finite element method is one of ways to solve problem in nature with numerical solution. Commonly, occurrence in nature could be explained by equation either differential or integral equation. Because of that reason, finite element method becomes one of ways to solve partial and integral differential form. Generally, finite element method enables users to get evolution in place or time from one or more than one variable representing physical system.

If referred to structure analysis, finite element method is a good method in calculating displacement, stress, and tenseness on a structure in pressuring [15].

Finite element method use is considered as one of numerical methods to solve some engineering problems which are very related to computer development with some other aspects like Computer Aided Design (CAD) and Computer Aided Engineering (CAE).

On its use, generally finite element method software has three main phases, which are as follows:

\section{1) Preprocessing}

In this phase, users make a model becoming a part to be analysed in which geometry is divided into discretized subparts or called "elementt". It is connected on discretization point namely node". Certain node will be set as fix displacement and the other part is set as load.

\section{2) Analysis}

In this phase, data inserted to preprocessing phase previously will be used as input on finite element code to develop and solve equation system of linier or non linier algebra.

$\mathrm{k} \cdot(\mathrm{x})=\mathrm{F}$

Where $\mathrm{x}$ and $\mathrm{F}$ are displacements and outer force given on a point. Matrix information of $\mathrm{k}$ depends on occurring problem type and module which will be directed to truss analysis approach and elastic linier stress. Paid software has more capacity to solve many problems.

\section{3) Post-processing}

It reveals end result after being analysed by module by showing displacements data and stress on discretized part in geometry model. Post-processor usually shows chart with colour contour displaying stress label occurring in geometry model [16].

There are finite element method software which are paid (commercial FEA software) and unpaid (free FEA software).

Element amount can influence error presentation which can be resulted from finite element method. The more element amount, the lower error presentation [17].

In this simulation, tooth is divided into 1667882 elements and 309356 nodal. To show stress data, points of the highest stress from simulation result will be taken.

\section{Theory}

In fact, angle configuration of arm, boom, and bucket in excavator are dynamic, so that its angle is always changed. However, it should not analyze every orientation or configuration of excavator. Thus, static configuration is set to obtain maximum power and then it will be used in this research [18].

\subsection{Bucket Curling Force (Fb)}

Based on SAE J1179: maximum radial force of bucket tooth was obtained from cylinder (bucket curling force) $F_{b}$. 
It is a digging force resulted from cylinder bucket and radius tangent $d_{D}^{1}$. Bucket should be positioned to get maximum moment output from bucket cylinder and connecting part. $F_{b}$ becomes maximum when $d_{A}$ range is maximum, because other ranges in equation (2) are constant [19].

$$
F_{b}=\frac{\text { Bucketcylinderforce }}{d_{D}}\left(\frac{d_{A} \times d_{C}}{d_{B}}\right)
$$

Bucket cylinder force $=($ working stress $) \times($ cylinder bucket longitudinal section $)$

If diameter of cylinder bucket piston $=D_{B}(\mathrm{~mm})$, working force is $P(\mathrm{MPa})$ and other ranges are in mm, then Equation 3 can be shown as follows:

$$
F_{b}=\frac{p \times\left(\frac{\pi}{4}\right) D_{B}}{d_{D}}\left(\frac{d_{A} \times d_{C}}{d_{B}}\right)
$$

\subsection{Bucket Crowd Force (Fs)}

Bucket crowd force $F_{s}$ is digging force resulted from arm cylinder and tangent radius $d_{f}$. Arm should be positioned to get maximum moment of arm cylinder and bucket. When calculating maximum value of $F_{S}$, arm cylinder center is in line with connecting line of pin cylinder arm and boom nose pin, then the equation is as follows:

$$
F_{S}=\frac{p \times\left(\frac{\pi}{4}\right) D_{A}^{2} \times d_{E}}{d_{F}}
$$

\subsection{Von Mises Stress Theory}

This theory assumes a failure conceded in friction stress which is bigger from what is assumed by maximum friction stress theory. For planning analysis, it is easier if von Mises stress is used, which is an equation connected with a stress in three axes.

$$
\sigma^{\prime}=\sqrt{\frac{1}{2}\left[\left(\sigma_{1}-\sigma_{2}\right)^{2}+\left(\sigma_{2}-\sigma_{3}\right)^{2}+\left(\sigma_{3}-\sigma_{1}\right)^{2}\right]}
$$

It will fail if: $\sigma^{\prime} \geq S_{y}$

From trials which have been done, it reveals that distortion energy theory (von Mises) assumes failure with the highest carefulness in all quadrants [20].

\section{Calculations}

Dimensional size of bucket model is as follows:

Table 4. Dimensional size of bucket and arm

\begin{tabular}{ccccccccc}
\hline $\mathbf{d}_{\mathbf{A}}(\mathbf{m m})$ & $\mathbf{d}_{\mathbf{B}}(\mathbf{m m})$ & $\mathbf{d}_{\mathbf{C}}(\mathbf{m m})$ & $\mathbf{d}_{\mathbf{D}}(\mathbf{m m})$ & $\mathbf{d}_{\mathrm{E}}(\mathbf{m m})$ & $\mathbf{d}_{\mathbf{f}}(\mathbf{m m})$ & $\mathbf{D}_{\mathbf{A}}(\mathbf{m m})$ & $\mathbf{D}_{\mathrm{B}}(\mathbf{m m})$ & $\mathbf{P}(\mathbf{M p a})$ \\
\hline 594 & 631 & 453 & 1496 & 829 & 4415 & 95 & 80 & 28,9 \\
\hline
\end{tabular}

Thus, loading value calculation like bucket curling force and bucket crowd force could be done by inserting dimensional data value in bucket to the formula.

\subsection{Bucket Curling Force (Fb) and Bucket Crowd Force (Fs)}

- $\mathbf{F}_{\mathbf{b}}$

$F_{b}=\frac{28.9 \times\left(\frac{\pi}{4}\right) 80}{1496}\left(\frac{594 \times 453}{631}\right)=41425.34752 \mathrm{~N}$

The numbers of tooth in bucket are 5, thus loading of $F_{b}$ accepted by tooth is $F_{b}$ divided by 5 :

$F_{b}=41425.34752 / 5=8285.06 \mathrm{~N}$ 
- $\mathbf{F}_{\mathrm{s}}$

$F_{s}=\frac{28.9 \times\left(\frac{\pi}{4}\right) 95^{2} \times 829}{4415}=38479.86374 \mathrm{~N}$

The numbers of tooth in bucket are 5, thus loading of Fs accepted by tooth is Fs divided by 5 :

$F_{S}=38479.86374 / 5=7695.97 \mathrm{~N}$

4.2. Force Vector

$F=8285.06 N$

$\frac{F_{x}}{F}=\cos 32^{\circ}$

$F_{x}=7026.12 \mathrm{~N}$

$\frac{F_{y}}{F}=\sin 32^{\circ}$

$F_{y}=4390.4 \mathrm{~N}$

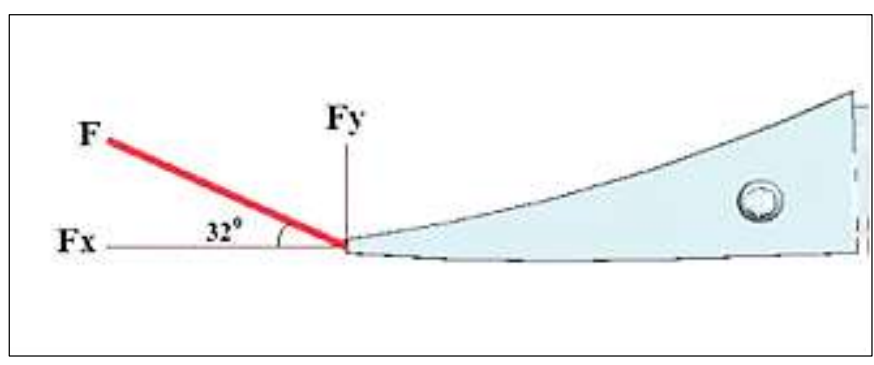

Figure 3. Diagram of free-pressuring object [5]

\subsection{Simulation with CAE Software}

After doing 3-dimension modelling in excavator tooth and getting pressuring process force value, then pressuring simulation is done by inserting needed parameters like force value, boundary condition, and properties material. These are the inserting processes of parameter value to CAE Software.

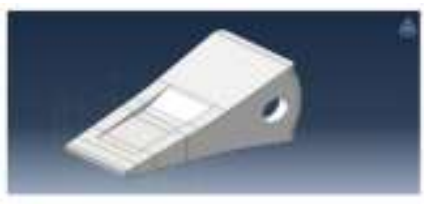

3-Dimension Plane

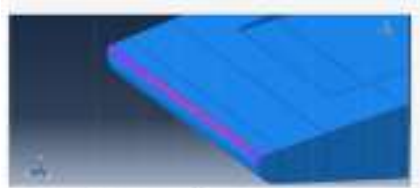

Coupling

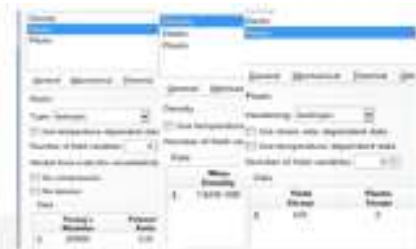

Properties Material

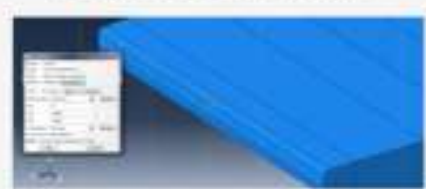

Force Load

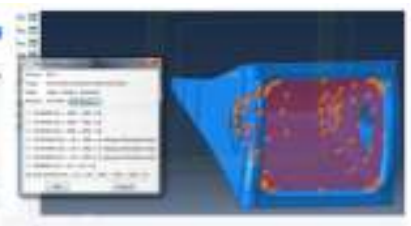

Boundary Condition

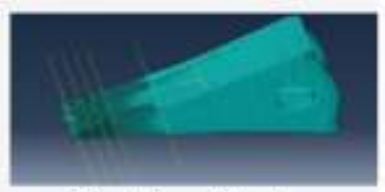

Meshing Part

Figure 4. Parameter insert on Abaqus 6.10

\section{Result and Discussion}

\subsection{Result}

After doing needed parameters inserting to simulation process, the next step is doing simulation process itself which 
is by calculating effective stress which is also known equivalent von Mises stress in every point occurring stress by using von Mises formula [21].

Loading is done by giving normal force on the end of bucket tooth which contacts with the soil when excavation. This analysis aims to know the magnitude of maximum stress of bucket tooth when doing penetration process toward soil. Therefore, it can be known whether there is a part of bucket tooth which fails or not. Maximum stress output occurring in bucket tooth is then compared to allowable stress of bucket tooth material. If maximum stress value of bucket tooth in simulation process is still below the allowable stress value of bucket tooth material, then it could be stated that bucket tooth form is safe. Simulation result is shown in form of von Mises stress in Figure 5.

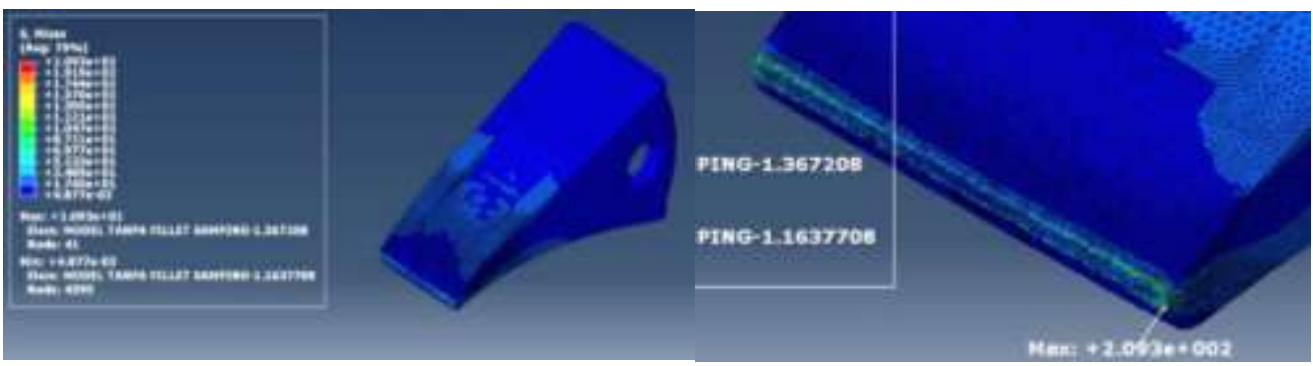

Figure 5. Von Misses stress value

While Figure 6 shows the deformation value result of bucket tooth in millimetre:

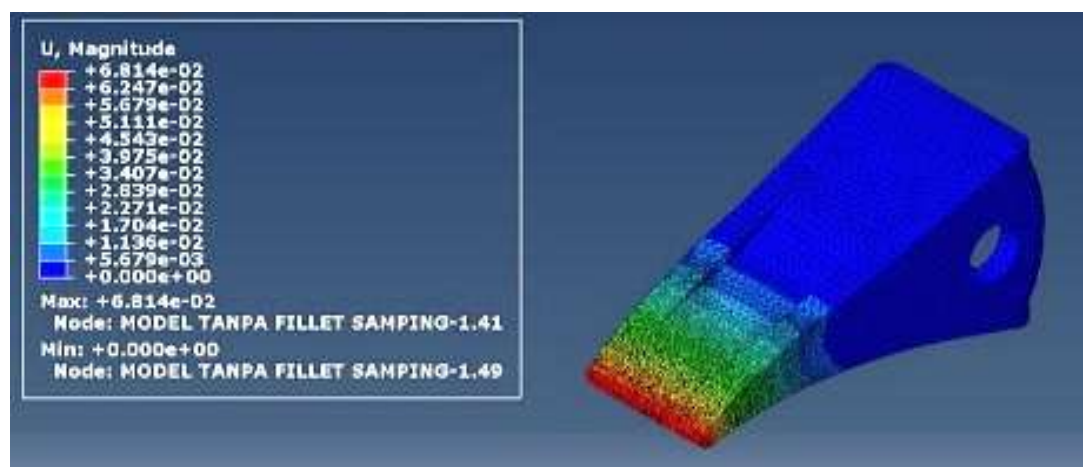

Figure 6. Deformation in bucket tooth

\subsection{Discussion}

Based on simulation result, it found different stress distributions. The part which gets force load has the highest stress. Maximum stress in bucket tooth of simulation result for force pressuring is in the end of bucket tooth which is in amount of 209.3 MPa. It shows that when bucket tooth is used continuously, then its both ends will get failure. The failure might be in form of wear, bent, crack and fracture during use. To prevent failure, hardening in bucket tooth ends is done.

The highest stress value of bucket tooth is in the part which has right angle. Curve form can decrease coefficient value of stress concentration. The higher radius value of curve, the lower coefficient value of stress concentration [22].

While, maximum deformation value of bucket tooth is $0.0681 \mathrm{~mm}$.

After getting maximum stress value of bucket tooth, then the result is compared to allowable stress from material of bucket tooth. Below is the calculation result of allowable stress of bucket tooth:

Safe Stress $=\frac{\text { Yield Strength }}{\text { Safety Factor }}=\frac{450}{2}=225 \mathrm{MPa}$

\section{Conclusion}

- From simulation result, it is found that maximum stress of bucket tooth is in its end part which has direct contact with soil. It can cause failure in bucket tooth end like wear, bent, crack and fracture during use.

- It is found that finite element method can be used for enhancing quality of in geometry form.

- The biggest force value resulted from excavator is obtained from calculation of bucket curling force. The magnitude of the highest force value which can be obtained is $8285.06 \mathrm{~N}$. 
- The highest stress value of bucket tooth is $209.3 \mathrm{MPa}$. This value is still below allowable stress value, so that bucket tooth form is considered safe.

- The biggest stress value of bucket tooth is on right angle.

- The biggest deformation value of bucket tooth is $0.0681 \mathrm{~mm}$ in the part which gets pressuring.

\section{References}

[1] Fernandez J.E., Vijande R., Tucho R., Rodriguez J., Martin A. Materials selection to excavator teeth in mining industry. Elsevier, Wear 250, pp. 11-18, 2001. Doi: https://doi.org/10.1016/S0043-1648(01)00624-X.

[2] Sanjay Kumar, D.P. Mondal, A.K. Jha, Effect of Microstructure and Chemical Composition of Hardfacing Alloy on Abrasive Wear Behavior, Journal of Materials Engineering and Performance, pp 649-655, 9(6) (2000). Doi: https://doi.org/10.1361/105994900770345511.

[3] GÁBOR LADÁNYI1, ISTVÁN SÜMEGI2 "Bucket and Cutting Tooth Developments for the Bucket Wheel Excavators of Mátra Power Station LLC", Annals of the University of Petroşani, Mechanical Engineering, pp 151162, Dec (2012).

[4] Y. Bayhan, Reduction of wear via hardfacing of chisel ploughshare, Tribology International, 39 (2006) 570-574. Doi: https://doi.org/10.1016/j.triboint.2005.06.005.

[5] Shaikh, B. P. and Mulla, A. M. "Analysis of bucket teeth of backhoe excavator loader and its weight optimization.” International Journal of Engineering Research \& Technology 4 (2015): 289-295.

[6] Deere, John. Bucket Tooth Catalogue. USA, 2002.

[7] A.Imam Wahyudi. Analisis Pengaruh Rake Angle Terhadap Distribusi Tegangan Pada Excavator Bucket teeth dengan Menggunakan Metode elemen Hingga. 2017.

[8] Fang ZQ, Hu GM, Gui WJ, Liu Y. Analysis of contact forces of particles with excavator bucket in digging process by using discrete element method. InMechanics and Mechanical Engineering: Proceedings of the 2015 International Conference (MME2015) 2016 Jul 14 (pp. 30-38). Doi: https://doi.org/10.1142/9789813145603_0004.

[9] Chen J, Zou Z, Pang X. Digging performance characterization for hydraulic excavator considering uncertainty during digging operation. Proceedings of the Institution of Mechanical Engineers, Part C: Journal of Mechanical Engineering Science. 2017. https://doi.org/10.1177/0954406217692843.

[10] Patel, Bhaveshkumar P. and Prajapati., J. M. "Evaluation of bucket capacity, digging force calculations and static force analysis of mini hydraulic backhoe." Jr. of Machine Design 4 (2012): 59-66.

[11] Zienkiewicz, O. C., Taylor, R. L. and Zhu, J. Z. The Finite Element Method: Its Basis and Fundamentals 6th Edition. Amsterdam, 2005.

[12] Singla, Shivali, Vineet Shibe, and J. S. Grewal. "Performance evaluation of hard faced excavator bucket teeth against abrasive wear using MMAW process." International Journal of Mechanical Engineering Applications Research 2, no. 02 (2011): $73-77$.

[13] Pollack, H.W. Materials Science and Metallurgy 2nd Edition. Singapore: McGraw-Hill, 1988.

[14] Fernandez, J. E., Vijande, R., Tucho, R., Rodriguez, J., and Martin, A. "Materials selection to excavator teeth in mining industry.' Elsevier, Wear 250 (2001): 11-18. Doi: https://doi.org/10.1016/S0043-1648(01)00624-X.

[15] Dagwar, K. S. Telrandhe, R. G. "Failure Analysis of Excavator Bucket Tooth." International Journal of Scientific Research and Engineering Studies 4 (2015): 2349-8862.

[16] Oñate, E. "Structural Analysis with the Finite Element Method." Linear Statics Lecture Notes on Numerical Methods in Engineering and Sciences. Barcelona: Artes Gráficas Torres S.A., 2003.

[17] Moaveni, S. Finite Element Analysis: Theory and Application with Ansys. Mankato: Prentice-Hall Inc., 1999.

[18] Liu, Y. “Choose the Best Element Size to Yield Accurate FEA Results While Reduce FE Model's Complexity" 1 (2013): $13-28$.

[19] SAE International. SAE J1179: Hydraulic Excavator and Backhoe Digging Force. Warrendale: SAE, 1990.

[20] Budynas, R. G., Nisbett, J. K., and Shigley, J. E. Shigley's mechanical engineering design. New York: McGraw-Hill, 2011.

[21] Virág, Z. \& Zirbik, S. "Examination of an Optimized Replaceable Cutting Tooth of Excavator.” Geosciences and Engineering, 1 (2012): 337-342.

[22] Zamma, A., Jalal, S.E., and Boubeker, B., "Elastic and Elasto-Plastic Study for Bucket Tooth - Contribution to the Increase for Resistance of Collapse." Advanced Materials Research, 682 (2013): 113-118. Doi: 10.4028/www.scientific.net/AMR.682.113. 